\title{
Crescimento e sobrevivência de larvas de peixe vermelho (Carassius auratus) alimentadas com microdietas comerciais
}

[Growth and survival of Carassius auratus larvae fed commercial micro diets]

\author{
P. Rema ${ }^{1,2}$, J. Martins ${ }^{1}$ \\ ${ }^{1}$ Departamento de Zootecnia - Universidade de Trás-os-Montes e Alto Douro \\ Apartado 1013 \\ 5001-801 - Vila Real, Portugal \\ ${ }^{2}$ Centro Interdisciplinar de Investigação Marinha e Ambiental - Porto, Portugal
}

\section{RESUMO}

Para avaliar o desempenho de dois alimentos comerciais para larvas de peixes ornamentais e analisar o efeito que a suplementação em vitaminas e minerais teria sobre a sobrevivência e o crescimento dessas, foi realizado um ensaio de crescimento de 21 dias, com 4800 larvas de peixe vermelho (Carassius auratus), distribuídas por 24 grupos (200 indivíduos por grupo). Cada grupo foi alojado em tanques de $5 \mathrm{~L}$ de capacidade, ligados a um sistema de recirculação de água. Quatro grupos foram alocados a um controle de jejum, em que os animais não foram alimentados, e os restantes 20 grupos foram utilizados em cinco tratamentos, em quadruplicado. Em cada um dos cinco tratamentos, os peixes ingeriram uma das seguintes dietas: $\mathrm{R}$ (referência), $\mathrm{C}_{1}$ (SERA vipan ${ }^{\mathbb{B}}$ baby), $\mathrm{C}_{2}$ (SERA ${ }^{\circledR}$ microgran), $\mathrm{C}_{1} \mathrm{v}$ e $\mathrm{C}_{2} \mathrm{v}\left(\mathrm{C}_{1}\right.$ e $\mathrm{C}_{2}$, respectivamente, reforçadas em vitaminas e minerais). As taxas de sobrevivência e crescimento dos peixes do tratamento $\mathrm{R}$ foram maiores que as obtidas nos outros tratamentos $(\mathrm{P}<0,05)$. Foram ainda observadas diferenças significativas entre tratamentos relativamente ao comprimento e peso totais. Face aos resultados observados, conclui-se que as duas dietas comerciais, com e sem suplementação, não parecem ser adequadas para o preenchimento das necessidades nutricionais de larvas de peixe vermelho.

Palavras-chave: Peixe vermelho, Carassius auratus, crescimento, sobrevivência microdietas

\begin{abstract}
A 21-day growth trial was performed to evaluate two ornamental fish larvae commercial diets and the effect of mineral and vitamin supplementation of the same commercial microdiets on growth and survival of goldfish larvae (Carassius auratus). A total of 4,800 larvae were randomly distributed in 24 groups of 200 individuals. Each group was housed in a $5 \mathrm{~L}$ tank, connected to a recirculating water system. Four groups were assigned to a fastening control. The animals in these groups did not receive any food. The remaining 20 groups were distributed in five treatments, in quadruplicate. The animals of each treatment were fed one of five experimental diets: $R$ (reference), $C_{I}$ (SERA vipan ${ }^{\circledR}$ baby), $C_{2}$ (SERA ${ }^{\circledR}$ microgran), $C_{1} v$, and $C_{2} v\left(C_{1}\right.$ and $C_{2}$, respectively, reinforced with minerals and vitamins). At the end of the trial, high survival and growth rates were observed in $R$ treatment. There were significant differences in total length and total weight among dietary treatments. Considering the high survival and growth rates of goldfish larvae obtained with the reference diet, it can be concluded that the two commercial diets tested, with or without mineral and vitamin supplements, do not seem suitable for feeding goldfish larvae.
\end{abstract}

Keywords: goldfish, Carassius auratus, growth, survival, microdiets

\section{INTRODUÇÃO}

A utilização de dietas adequadas à espécie e à fase do ciclo e vida em que se encontra é um dos

Recebido em 29 de dezembro de 2008

Aceito em 23 de abril de 2009

E-mail: prema@utad.pt fatores determinantes para o sucesso da produção de peixes em aquicultura, sejam eles destinados ao consumo ou ao ornamento (Kaiser et al., 2003; Lim et al., 2003). Entre os fatores mais importantes estão um correto equilíbrio entre os 
vários nutrientes, nomeadamente os teores em energia, proteína e a relação entre os dois, e os níveis de vitaminas, nomeadamente as vitaminas E e C. A vitamina E atua como um antioxidante, prevenindo a oxidação de colesterol e ácidos graxos poli-insaturados de fosfolipídios, nas membranas celulares e subcelulares (Nutrient..., 1983). Por outro lado, vários estudos evidenciaram o efeito que a vitamina $E$ pode ter sobre o crescimento e a sobrevivência de várias espécies de peixes, em várias fases do seu ciclo de vida. Entre estes, são de salientar os de Brown et al. (2005), Jalali et al. (2008) e James et al. (2008), os quais sugerem a utilização de níveis mais elevados do que aqueles que são correntemente praticados pela indústria de fabrico de alimentos compostos.

Outros estudos realizados em várias espécies (Ibiyo et al., 2006, 2007; Eo e Lee, 2008; Tewary e Patra, 2008) colocaram em evidência o efeito positivo que a suplementação em vitamina $\mathrm{C}$ tem sobre o crescimento, mais uma vez sugerindo a utilização de níveis superiores aos correntemente usados.

Deve ainda ser referido que o processamento de que o alimento for alvo e também o tipo de matérias-primas e o tratamento tecnológico a que estas foram sujeitas podem afetar a disponibilidade dos nutrientes presentes. Assim, a qualidade de um alimento vai depender em grande parte da qualidade dos seus nutrientes.

A indústria portuguesa de alimentos compostos para peixes não dispõe, até o momento, de dietas comerciais para carpa e muito menos para as larvas desse ciprinídeo. Este fato relaciona-se com as características da aquicultura portuguesa que não engloba a produção de carpas para consumo humano. Pelo contrário, o mercado de alimentos compostos para espécies de ornamento oferece uma extensa gama de produtos que incluem dietas para larvas de ciprinídeos.

No entanto, estes alimentos, além de apresentarem escassa informação sobre a composição química, possuem teores médios em minerais e em vitaminas ( 2 a 3\% da dieta) mais baixos do que aqueles que são correntemente utilizados em trabalhos de investigação científica sobre nutrição larvar em peixe vermelho e em carpas (Charlon e Bergot, 1984; Szlaminska et al., 1991; Bergot e Kestemont, 1995; Kaiser et al., 2003; Lim et al., 2003).

Neste experimento, pretendeu-se avaliar o efeito da utilização de duas microdietas comerciais, disponíveis no mercado da aquariofilia, no crescimento e na sobrevivência de larvas de peixe vermelho (Carassius auratus). Durante 21 dias, foi comparado o desempenho de larvas alimentadas com essas dietas comerciais (com ou sem suplementação em minerais, vitaminas e fosfolipídios) com aquele exibido por larvas idênticas, mas alimentadas com uma dieta de referência $(\mathrm{R})$.

\section{MATERIAL E MÉTODOS}

Foram utilizadas 4800 larvas de Carassius auratus que apresentavam peso e comprimento médios iniciais de $0,7 \pm 0,1 \mathrm{mg}$ e $5,1 \pm 0,3 \mathrm{~mm}$, respectivamente, determinados numa amostra de 150 larvas. Foram constituídos 24 grupos de 200 larvas (cinco dietas + controle de jejum, em quadruplicado), tal como referido por Rema e Gouveia (2005). O controle de jejum foi utilizado para averiguar a existência de outros nutrientes que não fossem os veiculados pelas dietas experimentais. A ser assim, deveria ocorrer a mortalidade completa dos grupos de controle no prazo de duas semanas, assegurando que a produtividade primária do volume de água em que o experimento decorreu não iria afetar os resultados globais obtidos.

Os restantes 20 grupos foram distribuídos por cinco tratamentos, a cada um correspondendo uma de cinco dietas experimentais: dieta referência (R), SERA vipan ${ }^{\circledR}$ baby $\left(\mathrm{C}_{1}\right)$, SERA ${ }^{\circledR}$ microgran $\left(\mathrm{C}_{2}\right), \mathrm{C}_{1}$ enriquecido $\left(\mathrm{C}_{1} \mathrm{~V}\right)$ e $\mathrm{C}_{2}$ enriquecido $\left(\mathrm{C}_{2} \mathrm{v}\right)$. $\mathrm{O}$ alimento $\mathrm{R}$ foi utilizado como dieta de referência e formulado de acordo com as recomendações de Bergot e Kestemont (1995), modificadas por Rema (2001). As dietas $\mathrm{C}_{1}$ e $\mathrm{C}_{2}$ são alimentos comerciais, correntemente utilizados no mercado da aquariofilia e destinados a alimentar larvas de peixes ornamentais. As dietas $\mathrm{C}_{1} \mathrm{v}$ e $\mathrm{C}_{2} \mathrm{v}$ correspondem às $\mathrm{C}_{1} \quad$ e $\quad \mathrm{C}_{2}$ adicionadas com pré-mistura de minerais e vitaminas, idêntica à utilizada na fabricação da R. Todas as dietas foram analisadas. A matéria seca (MS) foi determinada por secagem em estufa com ventilação forçada de ar, a $65^{\circ} \mathrm{C}$, até obtenção de peso constante; a proteína bruta $(\mathrm{PB} ; \mathrm{N} \times 6,25)$ foi determinada 
pelo método de Kjeldahl, de acordo com as normas ISO-5983 (1979); as cinzas foram determinadas após incineração em mufla a $600^{\circ} \mathrm{C}$ durante duas horas, segundo o método da AOAC (Official..., 1975); a matéria orgânica (MO) foi calculada pela diferença entre a MS e as cinzas; a gordura bruta $(\mathrm{GB})$ foi determinada por extração contínua com éter de petróleo, segundo a norma ASTN (Total..., 1988); a energia bruta (EB) foi determinada por combustão direta num calorímetro adiabático (PARR), a uma pressão de oxigênio de 30 atmosferas. Os ingredientes, a composição química e a composição das prémisturas mineral e vitamínica usados nos tratamentos são apresentados nas Tab. 1 e 2.

Para fabricar o alimento $\mathrm{R}$, a matéria-prima foi moída e peneirada até a obtenção de partículas com granulometria inferior a $100 \mu \mathrm{m}$. Aos ingredientes secos foi adicionada água até ser obtida uma pasta espessa. Esta foi posteriormente introduzida sob pressão num picador de carne (crivo de $3 \mathrm{~mm}$ ) para obtenção de um alimento úmido tipo spaghetti. Após secagem, em estufa ventilada a $40^{\circ} \mathrm{C}$, durante 48 horas, a dieta foi moída e calibrada em partículas de diferente tamanho: $<100,200,400$ e $630 \mu \mathrm{m}$. Finalmente, a microdieta foi embalada, referenciada e refrigerada $\left(4^{\circ} \mathrm{C}\right)$ para posterior utilização. Os alimentos $C_{1}, C_{2}, C_{1} V$ e $C_{2} V$ foram reduzidos a partículas de dimensões idênticas às do alimento $\mathrm{R}$ e processados de forma idêntica.

Os teores em minerais e em vitaminas dos alimentos comerciais $\mathrm{C}_{1}$ e $\mathrm{C}_{2}$ eram de $2 \%$ (dados do fabricante). Nos alimentos em que foi adicionada vitamina $\mathrm{C}$, esta foi incorporada separadamente, de acordo com as recomendações de Dabrowski et al. (1988) para larvas de Cyprinus carpio.

O ensaio de crescimento larvar foi realizado em uma bateria de 24 tanques, idêntica à descrita por Charlon e Bergot (1984), modificada por Rema (2001). No manejo dos animais e na distribuição do alimento, foram seguidos os procedimentos descritos por Rema (2001).

Tabela 1. Ingredientes e composição química das dietas utilizadas

\begin{tabular}{|c|c|c|c|c|c|}
\hline \multirow{2}{*}{ Ingredientes } & \multicolumn{5}{|c|}{ Alimentos experimentais } \\
\hline & $\mathrm{R}$ & $\mathrm{C}_{1}$ & $\mathrm{C}_{2}$ & $\mathrm{C}_{1} \mathrm{v}$ & $\mathrm{C}_{2} \mathrm{~V}$ \\
\hline Alimento comercial A & & 100 & & & \\
\hline Alimento comercial B & & & 100 & 90 & 90 \\
\hline Levedura protibel & 50 & & & & \\
\hline Fígado de bovino fresco & 34 & & & & \\
\hline Mistura vitamínica & 7 & & & 3 & 3 \\
\hline Mistura mineral & 5 & & & 3 & 3 \\
\hline Fosfatidilcolina $^{(1)}$ & 2 & & & 2 & 2 \\
\hline Vitamina C (25\%) & 1 & & & 1 & 1 \\
\hline Cloreto de colina & 1 & & & 1 & 1 \\
\hline $\begin{array}{l}\text { Composição } \\
\text { alimentos }^{(2)}\end{array}$ & & & & & \\
\hline Matéria Seca (MS) & 91,6 & 88,7 & 87,9 & 88,0 & 87,0 \\
\hline Proteína Bruta (\%MS) & 52,1 & 53,7 & 53,9 & 54,1 & 54,2 \\
\hline Gordura Bruta (\%MS) & 7,6 & 5,0 & 6,2 & 5,5 & 7,0 \\
\hline Energia Bruta $\left(\mathrm{kJ} \mathrm{g}^{-1} \mathrm{MS}\right)$ & 19,6 & 19,2 & 19,3 & 19,1 & 19,4 \\
\hline Vit. $\mathrm{E}\left(\mathrm{mg} \mathrm{kg}^{-1}\right.$ alimento) ${ }^{(3)}$ & 17500 & 200 & 200 & 2680 & 2680 \\
\hline Vit.C (mg kg-1 alimento) ${ }^{(3)}$ & 141 & 60 & 60 & 114 & 114 \\
\hline Cinzas $(\% \mathrm{MS})$ & 15,7 & 10,1 & 11,1 & 14,2 & 15,3 \\
\hline
\end{tabular}

${ }^{(1)} \mathrm{L}-\alpha$-lecitina $\left(60 \%\right.$ dos lipídios totais); ${ }^{(2)}$ cada valor é a média de 3 determinações; ${ }^{(3)}$ valores estimados ou indicados pelo fabricante.

Todos os valores são expressos em percentagem, exceto nos casos assinalados; Composição em minerais e em vitaminas dos alimentos comerciais $\mathrm{C}_{1}$ e $\mathrm{C}_{2}$ (por kg de alimento - declaração do fabricante): vit.A- 30 000UI; vit.B130mg; vit.B2- 90mg; vit.E- 60mg; vit.C- 200mg; vit.D3- 1500UI; cálcio- $17 \mathrm{~g}$; fósforo- $6 \mathrm{~g}$. 


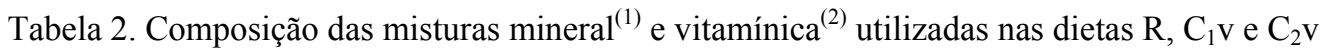

\begin{tabular}{|c|c|c|c|}
\hline \multicolumn{2}{|l|}{ Mistura vitamínica } & \multicolumn{2}{|c|}{ Mistura mineral } \\
\hline Vit. A (acetato a $500000 \mathrm{UI} \mathrm{g}^{-1}$ ) & 1,50 & Fosfato bicálcico & 500,00 \\
\hline Vit. D3 (100 000UI g $\left.{ }^{-1}\right)$ & 1,50 & Cloreto de cálcio & 215,00 \\
\hline Vit. E (ac. de tocoferol; 500UI g ${ }^{-1}$ ) & 6,00 & Cloreto de sódio & 40,00 \\
\hline Vit. K & 0,25 & Cloreto de potássio & 90,00 \\
\hline Tiamina (B1) & 1,00 & Carbonato de magnésio & 124,00 \\
\hline Rivoflavina (B2) & 2,50 & Sulfato de cobre & 3,00 \\
\hline Piridoxina (B6) & 1,00 & Sulfato de ferro & 20,00 \\
\hline Ácido nicotínico (B3) & 10,00 & Sulfato de zinco & 4,00 \\
\hline Ácido fólico (B9) & 0,50 & Fluoreto de sódio & 1,00 \\
\hline Vitamina B12 $\left(0,6 \mathrm{mg} \mathrm{g}^{-1}\right)$ & 2,50 & Sulfato de manganésio & 3,00 \\
\hline Inositol & 100,00 & Iodeto de potássio & 0,04 \\
\hline Biotina (B8) & 0,10 & Sulfato de cobalto & 0,02 \\
\hline Pantotenato de cálcio (B5) & 5,00 & & \\
\hline
\end{tabular}

$\mathrm{O} \mathrm{pH}$, a temperatura, o oxigênio dissolvido, a amônia, os nitritos e os nitratos foram controlados, com equipamentos digitais e testes colorimétricos, e registrados diariamente, tendo sido mantidos dentro dos valores recomendados para a espécie. O fotoperíodo pretendido foi assegurado por programadores, que também controlaram o funcionamento dos alimentadores automáticos Estes foram acoplados a motores elétricos específicos (Rema, 2001), o que, juntamente com a utilização dos programadores, permitiu dosar a quantidade e a frequência de distribuição de cada uma das microdietas experimentais.

Semanalmente, foi retirada uma amostra aleatória de 10 larvas de cada tanque que, depois de mortas por excesso de anestésico, foram conservadas em formol (10\%) para análise posterior. No final do experimento, foi efetuada uma contagem total das larvas sobreviventes. Os peixes desaparecidos foram considerados mortos e repartidos em função da mortalidade observada. Foram determinados os valores de sobrevivência $(\mathrm{S})$, comprimento médio $(\mathrm{mm})$, peso médio $(\mathrm{P} ; \mathrm{mg})$ e produto $\mathrm{PxS}$, entendido como critério global de comparação da biomassa final estimada dos diferentes lotes.

Para pesagem e medição, as amostras das larvas foram retiradas do formol e secas em papel absorvente, após o que os pesos foram obtidos em balança analítica de precisão $( \pm 0,0001 \mathrm{~g})$ e as medições obtidas por imagens fotográficas ${ }^{1}$

${ }^{1}$ Sony, CMSO, DSCR1 - Tóquio, Japão. digitalizadas, utilizando-se programa de análise de imagem ${ }^{2}$.

Os resultados são apresentados sob a forma de média \pm desvio-padrão. Os valores relativos à sobrevivência e ao peso médio larvar foram normalizados após transformação em $\operatorname{arc} \sin p^{1 / 2}$ e transformação logarítmica, respectivamente. A análise estatística foi feita utilizando o software $\mathrm{SAS} / 2008$. Após a análise da variância dos parâmetros estudados, a significância das diferenças das médias foi determinada por aplicação do teste Newman Keuls.

\section{RESULTADOS}

A taxa de sobrevivência variou entre 45,0 e $80,3 \%$. As larvas alimentadas com a dieta $\mathrm{R}$ apresentaram sobrevivência elevada e significativamente diferente $(\mathrm{P}<0,05)$ da observada nos restantes tratamentos (Tab. 3).

As larvas alimentadas com $\mathrm{C}_{1}$ e $\mathrm{C}_{2}$ registraram os valores mais baixos de sobrevivência $(50,4 \mathrm{e}$ $45,0 \%$, respectivamente). Estes alimentos, quando comparados com os seus homólogos enriquecidos, $\mathrm{C}_{1} \mathrm{~V}$ e $\mathrm{C}_{2} \mathrm{v}$, originaram taxas de sobrevivência significativamente mais baixas $(\mathrm{P}<0,05)$. Nos grupos de controle de jejum $(\mathrm{C})$, verificou-se uma taxa de mortalidade de 100\% após 14 dias de experiência, conforme previsto.

No final do ensaio, as larvas alimentadas com $\mathrm{R}$ cresceram significativamente mais $(P<0,05)$ que as alimentadas com as restantes dietas, não tendo sido verificadas diferenças significativas entre estas últimas.

${ }^{2}$ Image J; http://rsb.info.nih.gov/ij/ 
Tabela 3. Valores médios de sobrevivência (S), comprimento total (C), peso médio (P) e PxS das larvas de Carassius auratus, no final do ensaio (21 dias)

\begin{tabular}{|c|c|c|c|c|c|c|c|}
\hline & \multicolumn{6}{|c|}{ Tratamentos } & \multirow[b]{2}{*}{ EPM } \\
\hline & $\begin{array}{l}\text { Controle de } \\
\text { jejum }\end{array}$ & $\mathrm{R}$ & $\mathrm{C}_{1}$ & $\mathrm{C}_{2}$ & $\mathrm{C}_{1} \mathrm{~V}$ & $\mathrm{C}_{2} \mathrm{~V}$ & \\
\hline S (\%) & 0 & $80,3 a$ & $50,4 d$ & $45,0 \mathrm{e}$ & $78,1 b$ & $67,5 \mathrm{c}$ & 0,22 \\
\hline $\mathrm{C}(\mathrm{mm})$ & - & $14,2 \mathrm{a}$ & $9,3 \mathrm{~b}$ & $9,0 \mathrm{~b}$ & $9,3 \mathrm{~b}$ & $9,2 \mathrm{~b}$ & 0,37 \\
\hline$P(\mathrm{mg})$ & - & $63,5 \mathrm{a}$ & $7,3 \mathrm{c}$ & $6,4 d$ & $14,3 \mathrm{~b}$ & $13,2 b$ & 0,14 \\
\hline $\mathrm{P} \times \mathrm{S}$ & - & $51,0 \mathrm{a}$ & $3,7 \mathrm{~d}$ & $2,9 \mathrm{e}$ & $11,2 \mathrm{~b}$ & $8,9 \mathrm{c}$ & \\
\hline
\end{tabular}

Valores são médias (10 larvas $x 4$ tanques). EPM: erro-padrão da média.

Valores na mesma linha seguidos por letras distintas diferem entre si $(\mathrm{P}<0,05)$.

O peso médio final das larvas alimentadas com os regimes constituídos pelas dietas comerciais suplementadas foi significativamente mais elevado $(\mathrm{P}<0,05)$ que o verificado nas larvas que receberam as dietas sem suplemento. No entanto, as dietas comerciais, suplementadas ou não, proporcionaram pesos finais significativamente mais baixos $(\mathrm{P}<0,05)$ face aos observados nas larvas alimentadas com R. O melhor resultado para PxS verificou-se também no grupo de larvas alimentadas com R. Este valor foi significativamente mais alto $(\mathrm{P}<0,05)$ que o observado em todas as outras dietas. As dietas comerciais suplementadas com minerais e vitaminas provocaram uma ligeira, em termos numéricos, mas significativa melhoria na biomassa produzida relativamente às dietas não suplementadas.

\section{DISCUSSÃO E CONCLUSÕES}

As larvas alimentadas com a dieta apresentaram performance produtiva elevada, semelhante ao observado por outros autores (Charlon e Bergot, 1984; Charlon et al., 1986; Alami-Durante et al., 1991; Rema, 2001; Rema e Gouveia, 2005). Este fato confere ao alimento composto utilizado uma qualidade nutricional elevada para larvas de Carassius auratus. Pelos resultados observados, as comerciais não cobrem completamente as necessidades nutricionais das larvas e, portanto, não parecem ser as mais indicadas para a obtenção de bons resultados de produção, pelo menos para as larvas da espécie em estudo.

$\mathrm{O}$ peso médio final das larvas alimentadas com $\mathrm{R}$ foi mais baixo $(63,5 \mathrm{mg})$ quando comparado com os obtidos em experiências anteriores (Rema, 2001; Rema e Gouveia, 2005), em que foram observados valores médios de $105,0 \mathrm{mg}$. Este fato se refletiu de forma evidente na biomassa produzida. A diferença observada está, muito provavelmente, relacionada com peso e comprimento médios iniciais das larvas e com os valores da temperatura da água. Em ambos os casos, os valores foram mais baixos do que os utilizados em ensaios prévios (Kestemont, 1995; Rema, 2001; Rema e Gouveia, 2005).

Pelos teores de PB e EB das dietas comerciais utilizadas, pode-se afirmar que estes indicadores brutos não foram os entraves ao crescimento das larvas. As limitações dessas dietas são, provavelmente, a qualidade da proteína (perfil e disponibilidade de aminoácidos essenciais) e/ou a disponibilidade da EB.

A suplementação das dietas comerciais com minerais, vitaminas, fosfatidilcolina e colina resultou em acréscimos significativos na sobrevivência e no peso das larvas em relação às não suplementadas. Este efeito, no entanto, foi mais visível na sobrevivência larvar, já que não foi observado efeito sobre o comprimento. Quanto ao peso, as larvas alimentadas com as dietas suplementadas não atingiram mais do que um quinto do peso daquelas alimentadas com $R$. Isto pode significar que os teores de minerais e de vitaminas utilizados nas dietas comerciais para peixes de ornamento, apesar de mais baixos que os recomendados por Bergot e Kestemont (1995) para larvas de carpa, provavelmente não foram os fatores responsáveis pelo baixo peso atingido pelas larvas neste estudo.

Segundo Geurden et al. (1993, 1995), Kanazawa (1994) e Radunz-Neto et al. (1994), as necessidades em fosfolipídios são elevadas nas fases larvares e, assim, a sua incorporação à dieta favorece à sobrevivência, ao contrário do verificado com os suplementos em ácidos graxos essenciais da série 13 (Radunz-Neto et al., 1994). Esse fato e os efeitos das vitaminas $E$ (James et al., 2008) e C (Dabrowski et al., 1988), 
podem ter influenciado na sobrevivência e no crescimento das larvas.

Os resultados obtidos devem servir apenas como meros indicadores e não devem ser extrapolados para a generalidade dos alimentos comerciais para peixes de ornamento. Neste estudo, foram utilizadas apenas duas dietas comerciais que fazem parte de um universo de alimentos para peixes ornamentais, muito vasto e difícil de tipificar. Nesse contexto, o delineamento de trabalho experimental suplementar deverá contribuir para a formulação de alimentos compostos nutricionalmente mais equilibrados, que promovam crescimentos elevados e taxas de sobrevivência larvares mais favoráveis nas espécies de ornamento.

\section{REFERÊNCIAS BIBLIOGRÁFICAS}

ALAMI-DURANTE, H.; CHARLON, N.; ESCAFFRE, A.M. et al. Supplementation of artificial diets for common carp (Cyprinus carpio L) larvae. Aquaculture, v.93, p.167-175, 1991.

BERGOT, P.; KESTEMONT, P. L'alimentation artificielle des larves de carpe. In: BILLARD, R. (Ed). Les carpes: biologie et élevage. Paris: INRA, 1995. p.164-168.

BROWN, M.R.; DUNSTAN, G.A.; NICHOLS, P.D. et al. Effects of a-tocopherol supplementation of rotifers on the growth of striped trumpeter Latris lineata larvae. Aquaculture, v.246, p.367-378, 2005.

CHARLON, N.; BERGOT, P. Rearing system for feeding fish larvae on dry diets - Trial with carp (Cyprinus carpio L) larvae. Aquaculture, v.41, p.1-9, 1984.

CHARLON, N.; DURANTE, H.; ESCAFFRE, A.M. et al. Alimentation artificielle des larves de carpe (Cyprinus carpio L). Aquaculture, v.54, p.83-88, 1986.

DABROWSKI, K.; HINTERLEITNER, S.; STURMBAUER, C. et al. Do carp larvae require Vitamin-C? Aquaculture, v.72, p.295-306, 1988.

EO, J.; LEE, K.-J. Effect of dietary ascorbic acido on growth and non-specific immune responses of Tiger puffer, Takifugu rubripes. Fish Shellfish Immunol., v.25, p.611-616, 2008.

GEURDEN, I.; RADUNZ-NETO, J.; BERGOT, P. Essentiality of dietary phospholipids for carp
(Cyprinus carpio L.) larvae. Aquaculture, v.131, p.303-314, 1995.

GEURDEN, I.; RADUNZ-NETO, J.; BERGOT, P. Response of carp (Cyprinius carpio) larvae to dietary phospholipids. In: INTERNATIONAL CONFERENCE WORLD AQUACULTURE '93, 1993, Torremolinos, Spain. Abstracts... Torremolinos: CWA, 1993. p.226.

IBIYO, L.M.O; ATEH, J.O.; OMOTOSHO, J.S. et al. Possible improvement in income with vitamin $\mathrm{C}$ fortified diets in practical farming of Heterobranchus longifilies fingerlings. J. Anim. Vet. Adv., v.6, p.479-484, 2007.

IBIYO, L.M.O.; MADU, C.T.; EZE, S.S. Effects of vitamin $\mathrm{C}$ supplementation on the growth of Heterobranchus longifilies fingerlings. Arch. Anim. Nutr., v.60, p.325-332, 2006.

ISO 5983:1979: Animal feeding stuffs. Determination of nitrogen content and calculation of crude protein content. Geneva: ISO, 1979.

JALALI, M.A.; HOSSEINI, S.A.; IMANPOUR, M.R. Effect of vitamin E and highly unsaturated fatty acid-enriched Artemia urmiana on growth performance, survival and stress resistance of Beluga (Huso huso) larvae. Aquac. Res., v.39, p.1286-1291, 2008.

JAMES, R.; VASUDHEVAN, I.; SAMPATH, K. Effect of dietary vitamin $\mathrm{E}$ on growth, fecundity, and leucocyte count in Goldfish (Carassius auratus). Isr. J. Aquac. Bamidgeh, v.60, p.121-127, 2008.

KAISER, H.; ENDEMANN, F.; PAULET, T.G. A comparison of artificial and natural foods and their combinations in the rearing of goldfish, Carassius auratus (L.). Aquac. Res., v.34, p.943950, 2003.

KANAZAWA, A. Essencial phospholipids of fish and crustaceans. In: KAUSHIK, S.J.; LUQUET, P. (Ed.). Fish nutrition in practice. Paris: INRA, 1994. p.519-530. (Les Colloques).

KESTEMONT, P. Influence of feed supply, temperature and body size on the growth of goldfish Carassius auratus larvae. Aquaculture, v.136, p.341-349, 1995.

LIM, L.C.; DHERT, P.; SORGELOOS, P. Recent developments in the application of live feeds in the freshwater fish culture. Aquaculture, v.227, p.319-331, 2003. 
LUQUET, P. Efficacité des proteínes en relation avec leur taux d'incorporation dans l'alimentation de la truite arc-en-ciel. Ann. Hydrobiol., v.2, p.175-186, 1971.

NUTRIENT requirement of warmwater fishes and shell fishes. Washington: National Academy of Sciences, 1983. 102p.

OFFICIAL methods of analysis of the AOAC. 12.ed. Washington: AOAC, 1975.

RADUNZ-NETO, J.; CORRAZE, G.; CHARLON, N. et al. Lipid supplementation of casein-based purified diets for carp (Cyprinus carpio L) larvae. Aquaculture, v.128, p.153-161, 1994.

REMA, P. Estudos de nutrição em ciprinídeos ornamentais. 2001. 194f. Dissertação (Doutoramento) - Universidade de Trás-osMontes e Alto Douro, Vila Real, Portugal.
REMA, P.; GOUVEIA, A. Growth and survival of goldfish (Carassius auratus) larvae reared at different densities. J. Anim. Vet. Adv., v.4, p.163166, 2005.

SALMON and trout feeding. Roma: FAO, 1971. 125p. (EIFAC Technical Papers, 12).

SZLAMINSKA, M.; ESCAFFRE, A.M.; BERGOT, P. Utilization of dietary pregelatinized starch by common carp (Cyprinus-Carpio L) larvae. J. Anim. Physiol. Nutr., v.65, p.65-71, 1991.

TEWARI, A.; PATRA, B.C. Use of vitamin C as an immunostimulant. Effect on growth, nutritional quality, and immune response of Labeo rohita (Ham.). Fish Physiol. Biochem., v.34, p.251-259, 2008.

TOTAL fat extraction in certain food products according to AOAC: Application short note. Höganäs, Sweden: Tecator, 1988. 